\title{
University students' mental construction when learning the Convergence of a Series concept
}

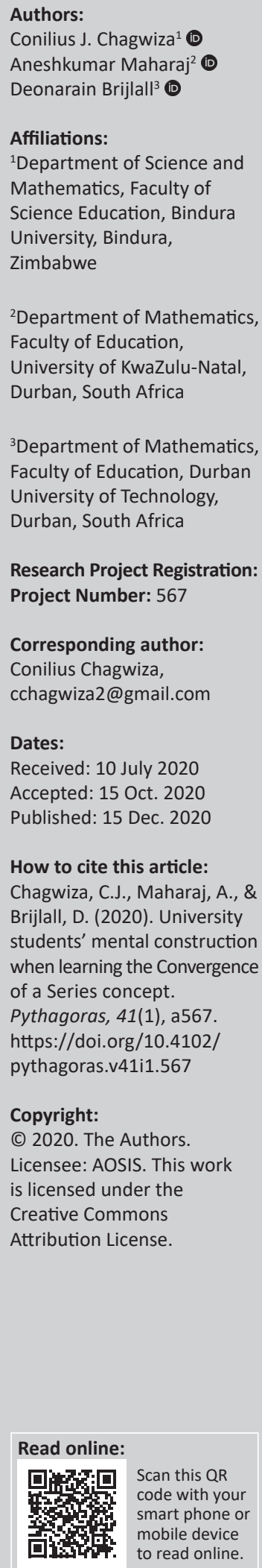

This article uses action-process-object-schema theory to study the mental constructions about the limit of series during a Calculus 1 course at a university. The researchers also used the theory's teaching methodology to teach the topic. A plethora of research on the limit concept is available and suggests that the concept is on record as being difficult for students to learn and comprehend. The study proposed a genetic decomposition on how undergraduate students might demonstrate their mental constructions in learning the limit of a series. Students were taken through the activities-classroom discussions-exercises cycle. Thirty students participated in answering questions based on the convergence of a series. The students' written responses together with the interviews were analysed and based on the findings a revision of the preliminary genetic decomposition was done. We found that there were students who did not display the predicted mental construction indicated by the preliminary decomposition in the application of the definition for the convergence of a series, but displayed the predicted mental construction for the application of the series convergence tests. We also found that certain schema were necessary for the achievement of a complete understanding of the convergence of a series concept. The mental constructions within the missing schema were included in the modified genetic decomposition. This empirically enriched model is now expected to inform pedagogy on the convergence of a series concept.

Keywords: action; process; object; schema; limit; series.

\section{Introduction}

Many mathematical concepts in calculus and other courses depend heavily on the limit concept, like the definite integral as the limit of Riemann sums, Taylor series and the differential in multivariate calculus. Convergent partial sums of a sequence may be used to define the limit of an infinite series. The limit of an infinite series can be defined as the limit (as $n \rightarrow \infty)$ of the sequence of partial sums. Infinite series development was motivated by the approximation of unknown areas and for the approximation of the value of $\pi$ (Hartman, 2008). In about 1350, Suiseth indicated that $\frac{1}{2}+\frac{2}{2^{2}}+\frac{3}{2^{3}}+\ldots \ldots+\frac{k}{2^{k}}+\ldots=2$ (Stillwell, 1989). Madhava (1340-1425) used $\frac{\pi}{4}=1-\frac{1}{3}+\frac{1}{5}-\frac{1}{7}+\ldots$ to estimate $\pi$ and $\sqrt{12}\left(1-\frac{1}{3.3}+\frac{1}{5.3^{2}}-\frac{1}{7.3^{3}}+\ldots\right)$ for $\pi$ (Joseph, 2000).

Our experiences suggest that infinite series is one of those topics that many students do not appreciate. They are unable to connect infinite series application outside their calculus class which makes them believe that the infinite series application is not useful in their lives. However, infinite series play a vital part in the field of ordinary differential equations and in the field of partial differential equations. Infinite series is an important aspect of calculus, Riemann sums, and sequences and series. The infinite sums and series can be 'well' or 'poorly' behaved. For example, if one is required to find the sum of $1+2+3+4+5+\ldots$, which is a 'well'-behaved sum, one can add the numbers and get an ever-increasing sum. The sum of $1-1+1-1+1-1+\ldots$ represents an example of a 'poorly' behaved sum, since for two consecutive terms, the sum is zero. Furthermore, technology can be used to discover convergence of series and their generation. Technology can also be used to create symbolic, numerical and graphical representations, and to change among these different illustration methods.

The purpose of this study was to explore the understanding of the limit of series using action-process-object-schema (APOS) theory, for 30 students who had registered for a calculus course. This study aimed to add to the body of research on limits of series. Furthermore, it suggested an alternative method for the learning of limits in relation to series in universities by proposing a genetic decomposition (GD) for the concept of a limit of an infinite series. We also use this proposed 
GD to explore the mental construction displayed by undergraduate students when dealing with the concept of a limit of an infinite series. The purpose of such an analysis was to observe whether our proposed GD was an accurate predictor of undergraduate students' mental constructions and, if not, then what modifications were required in the proposed GD. In order to achieve this purpose, we formulated the following research questions: What mental constructions do undergraduate mathematics students reveal when solving problems involving the convergence of a series concept? How can these identified mental constructions of participating undergraduate mathematics students be used to refine the proposed GD?

It was hoped that the answers to these two research questions would provide a model that will inform mathematics educators on the various mental constructions students possess when dealing with the concept of a limit of an infinite series. Also, mathematics educators could use the modified GD, which was empirically developed in this study, to design questions for instruction, consolidation and assessment. This contribution to the field of the learning of undergraduate mathematics is one of the strong traits of APOS theory. Similar studies effectively formulated the modified GDs: Brijlall \& Ndlazi (2017) explored preservice teachers' mental constructions of the concepts of injections, surjections and bijections and in another study (Brijlall \& Ndlazi, 2019) presented a modified GD for integration techniques.

\section{Literature review}

Researchers have noted that there is a lack of research on the learning and teaching of infinite series (Earls, 2017; Earls \& Demeke, 2016). It is known from the researchers' experiences that a source of cognitive difficulties for students is the limit concept (Denbel, 2014). Many students struggle with limits (Cappetta \& Zollman, 2013; McCombs, 2014; Patel, McCombs \& Zollman, 2014). Those researchers identified specific student difficulties with limits. Cappetta and Zollman (2013) found that students have difficulties with understanding limits, involving the infinite processes of limits and the value of limits. Additionally, metaphorical reasoning, a way of understanding a situation to resolve disequilibrium that students encounter when faced with a new problem situation (Patel et al., 2014), develops without the knowledge of the lecturer and impedes students' understanding of mathematical concepts. A student who understands a mathematical concept can move between numerical, algebraic, graphical and application representations. However, most students compartmentalise their thinking resulting in them staying with procedures even if such procedures result in illogical or contradicting results (McCombs, 2014). Gulcer (2012) argues that even if lecturers move smoothly between limit as a number (end state) and as a process, students seem to focus only on limits as a process. Further, strong students can view limits as both a value (a static end state) and as a dynamic process (never-ending). Thus, the documented literature testifies to the lack of research on the limit concept in relation to series.
Misconceptions could be deeply ingrained in the mental map of an individual. Some students hold the misconception that the limit of the sequence of partial sums and the sum of an infinite series are not the same. Some students tried to determine the sum of the series by first adding all the terms (or as many as one could to determine a pattern) and then took the limit of the partial sums (Martínez-Planell, Gonzalez, DiCristina, \& Acevedo, 2012). Martínez-Planell et al. (2012) noticed in general that students relied on properties regarding finite sums, rather than looking at the limit of the sequence of partial sums or limit of the nth partial sum. Furthermore, Nardi and Iannone (2001) discovered that students had difficulty accepting that the convergence tests can be inconclusive.

Earls (2017) carried out a study on students' misconceptions on sequences and series, for second semester calculus. The main findings and some implications from that study were:

- When using the ratio test, some students got a value of 1 , and concluded that the series converged to 1 . Such students do not know that the meaning of a value of 1 in the ratio test is that the test is inconclusive with respect to possible convergence of the series.

- When determining which series convergence test was to be used for a given problem, some students faced difficulties in selecting the appropriate test. The difficulties of such students include the identifying of an infinite geometric series, which results in them using a root test to determine the convergence of a given infinite (geometric) series.

- Some students had trouble identifying the contrapositive of the nth term test and the logical equivalence of a statement. The implication here is that students require a greater exposure to these in the context of focused problems.

- Distinguishing between the limit of a sequence and the sum of a series was also a challenge for some students. In particular students had difficulty with regard to the difference between a sequence of numbers and a sequence of partial sums.

- Some students had difficulties with the limit and series notations, and their interpretations.

- When applying tests for convergence some students failed to check that the assumptions were satisfied. In particular this was noted when applying the integral and direct comparison tests.

- Students had misconceptions about what could be concluded from the results of the convergence tests. There were several examples of students who thought that a series test would give the sum of the series, rather than just whether the series converged.

Each of the above findings has teaching and learning implications. These were taken into consideration by the researchers of the current study when they designed the questions and problems for the participating students.

The study reported on in this article contributes to the limited literature on research related to the limit concept in the 
context of series. It also provides a proposed GD and modified GD, based on APOS theory, which mathematics educators could use to design questions for instruction, consolidation and assessment. Further, it gives an insight into the types of mental constructions that need to be focused on during the teaching and learning process.

\section{Theoretical framework}

The APOS framework describes the hierarchical developmental growth in understanding of mathematics concepts through mental constructions, namely action, process, objects and schema. APOS theory was developed from the work of Piaget and constructivist ideas (Arnon et al., 2014). The theory is centred on the models of what might be taking place in the mind of a university student as they engage with mathematical concepts such as limit of a series. It comprises general descriptions of the mental structures and mental mechanisms (Arnon et al., 2014).

As actions are repeated and reflected on, students move from relying on external cues to having internal control over them. This is categorised by an ability to imagine carrying out the steps without necessarily having to perform explicitly each one, thus showing the ability to skip steps, as well as reversing them. This mental shift is made possible by the interiorisation mechanism. Encapsulation occurs when the individual applies an action to a process; that is, the individual student sees a process as a static structure to which actions can be applied. When a process has been encapsulated into a mental object, it can be de-encapsulated back to its underlying process when the need arises. Furthermore, the mechanism of coordination is indispensable in the construction of some objects. Dubinsky and McDonald (2002) define the components of APOS theory as follows:

- Actions: An action is transformation of objects perceived by the individual as essentially external and as requiring, either explicating or from memory, stepby-step instructions on how to perform the operation. An example of this is when students are required to determine whether the series $\sum_{i=1}^{\infty} i$ is a convergent one. If the individual works $S_{1}$ by substitution and then $\mathrm{S}_{2}, \mathrm{~S}_{3}$, etc. then at each partial sum the individual demonstrates they are operating at an action conception.

- Process: This is when an action is repeated and the individual reflects upon it so that they can think of performing the same kind of action, but no longer with the need of external stimuli. Suppose the student wants to determine the convergence of a series say, $\sum_{i=1}^{\infty} i$ and calculates the nth partial sum and avoids calculating individual partial sums. Such a student has interiorised the procedure for finding out whether a series is convergent or not.

- An object: This is described as what is formed from a process. An individual becomes aware of the process as a totality and realises that transformations can act on it.
An illustration of this is when a student can perform an action on the process conception of the monotonic increasing or continuity notions when applying convergence tests.

- A schema: This is an individual's collections of actions, processes, objects and other schemas, which are linked by some general principles to form a framework in the individual's mind that may be brought to bear upon problem situations involving related concepts. In our case we can speak of an infinite series schema.

The major tool used in APOS-based research is a GD, which is a hypothetical model of mental constructions that a student may need to make in order to learn a mathematical concept (Arnon et al., 2014). A GD is a hypothesis that needs to be tested experimentally and is referred to as preliminary genetic decomposition (PGD; Arnon et al., 2014).

\section{Preliminary genetic decomposition for limit of a series}

The specific mental constructions relating to concepts of limit of series are detailed below. We drew upon the discussion by Arnon et al. (2014, p. 51) on examples of what a GD is not, to refine our GD. This we did to avoid the common errors that can confound a sound description of a GD with description of teaching sequence or mathematical description of a concept.

The expected APOS level for the limit of a series are shown in the PGD in Table 1.

TABLE 1: Preliminary genetic decomposition of the limit of a series.

\begin{tabular}{|c|c|}
\hline Level & Expected competence \\
\hline Action & $\begin{array}{l}\text { The individual is able to give examples of convergent series, state } \\
\text { the definition of convergence of an infinite series in terms of a limit } \\
\text { of an infinite sequence, make use of some explicit algorithm which } \\
\text { is seen by the individual as externally driven, for example finding } \\
\text { partial sums by substitution. }\end{array}$ \\
\hline Process & $\begin{array}{l}\text { The individual is able to find out about the convergence of a given } \\
\text { series by working out the limit of the sequence of the partial sums } \\
\text { without calculating each term of the series before determining the } \\
\text { limit of the sequence of partial sums. At this level, the individual is } \\
\text { able to predict whether the series converges or diverges by looking } \\
\text { at its structure. using the definition of convergence of an infinite } \\
\text { series to evaluate limits of the sequence of the partial sums by } \\
\text { showing the ability to coordinate actions to come up with } \\
\text { responses. For example, to find out about the convergence of the } \\
\text { series } \sum_{n=2}^{\infty} \frac{1}{n^{2}-1} \text { the individual forms the partial sum and } \\
\text { investigates its convergence. }\end{array}$ \\
\hline Object & $\begin{array}{l}\text { The individual is able to determine convergence or divergence of } \\
\text { an infinite series using series tests without detailed explanations, } \\
\text { for example when finding the limit of the series } \sum_{n=1}^{\infty} \frac{n^{2}+2}{n^{4}+5} \text {. } \\
\text { Actions (the convergent tests) are performed on a process } \\
\text { (relevant features of the terms of the given series) and the } \\
\text { subject must encapsulate it to become a total entity. A student } \\
\text { must already have a process understanding of both the } \\
\text { convergence of an infinite series concept and a process } \\
\text { understanding of the various series convergence tests. Then the } \\
\text { student needs to act on the process conception of relevant } \\
\text { features of the terms of the series when ensuring the hypotheses } \\
\text { of the convergence tests are applicable. In this case the student } \\
\text { will demonstrate an object conception of the notion of the } \\
\text { convergence of an infinite series. }\end{array}$ \\
\hline Schema & $\begin{array}{l}\text { The individual shows the ability to link all actions, processes and } \\
\text { objects into a coherent whole when investigating solutions to tasks } \\
\text { related to the convergence or divergence of infinite series. }\end{array}$ \\
\hline
\end{tabular}




\section{Methodology}

This section focuses on research design, participants, ethical issues, implementation of instruction and tasks.

\section{Research design}

In this study, we used the interpretive research paradigm as it recognises that individuals with varied backgrounds and experiences contribute to the ongoing construction of reality in their context (Wahyuni, 2012). The interpretive paradigm assumes that for researchers to understand some phenomena they should use the participants' understanding of the limit of a series concepts. This study focused on gaining a deeper understanding of the university students' understanding of limit of a series.

\section{Participants}

The participants were students from a Zimbabwean university who were first introduced to series in high school mathematics. However, in high school, formal definition of a limit of a series is not covered. In this study, all the 30 firstyear university students volunteered to participate. There were 4 mathematics major and 26 statistics and financial mathematics major students who took part in this study. All 30 students attempted the tasks and their written responses were analysed and summarised in Table 2. Since this qualitative study could not present $30 \times 7$ written responses we decided to choose written responses representing the students' display of a particular mental construction. We counted the number of responses that displayed the mental construction and those who did not. Those who did not were categorised as N. For each of the seven tasks we adopted purposive sampling and chose an attempt we thought demonstrated the particular mental construction (as predicted by the PGD) and one from the $\mathrm{N}$ category. In this way we identified eight students who were then interviewed, to clarify our initial judgements. Note that some students were interviewed more than once: for task 1a (ST15 and ST20), for task 1b (i) (ST28 and ST17), for task 1b (ii) (ST20 and ST13), for task 1b (iii) (ST12 and ST28), for task 1c (i) (ST28 and ST17, for task 1c (ii) (ST28 and ST17) and for task 1c (iii) (ST1 and ST14).

\section{Ethical issues and criteria for evaluation of qualitative research}

For the sake of unrecognisability, the students were coded using tags 'ST1', 'ST2', up to 'ST30'. The order did not carry any implication. While enabling the organisation of data, the codes ensured that the responses could not be linked in any way in the publication of results to the original participant. To ensure that the data accurately reflected students' thinking, a number of measures were taken. Informed consent forms were given to all participants, and the researcher read and clarified its contents. Participants were assured that their responses would firmly be preserved confidentially and that the data so gathered were for the use of the study only. It was communicated to the students that participation was completely voluntary, and that one could withdraw their services at any stage if they wished to do so. The researcher also outlined the nature, purpose and procedure of the study to the participants. Further, the researcher clarified the participants' concerns during the course of the study, whenever they arose.

In addition to research ethics we considered the criteria for scientific rigour in so far as qualitative research is concerned. Bitsch (2005) provided techniques to ensure credibility. For our study we resorted to persistent observation. Through the activities-classroom discussions-exercises (ACE) cycle students were first afforded the instruction on the convergence of an infinite series concept, then responded to seven tasks that addressed the PGD and later a selected group of students were interviewed to verify the researchers' judgement of the mental constructions the students displayed. In this way triangulation was addressed. In order to satisfy persistent observation (Bitsch, 2005) we carried out in-depth analysis to gain detail of the mental constructions demonstrated by the students. For transferability the modified GD will contribute to APOS theory and pedagogy on the convergence of infinite series. In order to remove subjectivity of the researchers we carried out interviews with students with written responses that depicted the category of mental construction achievement. In this way we sought conformability by the removal of bias and prejudices.

\section{Implementation of instruction}

The ACE learning cycles were used in this research. The activities were done through the use of Maple, a computer software or Computer Algebra System (CAS) which is able to solve the problems on limits of series in numerical and symbolic form. It was used during the learning phase in this study. Maple provides users with tools that are very easy to operate and are contained in palettes. Palettes are used to simplify writing in worksheets. Symbol palettes are used to write mathematical symbols, expression palettes are used to facilitate writing of mathematical expressions such as integrals, Sigma series and root forms. The program allows students of different computer skills to work fluently (Samkova, 2012). Teaching calculus using a variety of computer facilities equipped with Maple software facilitated lecturers to deliver material quickly, and students took lessons directly with practice. The learning phase included the use of computers, using Maple.

\section{Tasks}

The tasks (questions) were designed in line with the PGD. For the action conception we asked questions on recall of definition of convergence of an infinite series and the determination of the convergence of a particular infinite series by calculating individual successive terms of the series. To retrieve understanding on the process conception of the students' mental constructions we asked students to prove convergence using the definition of convergence of an infinite series. This required students to apply the definition of convergence. An individual will be required to form the 
sequence of the partial sums. Thereafter we asked the students to test specific series by using convergence or divergence tests. We thought that the student firstly would be expected to have a process conception of the features of the terms of the series in order to apply a specific convergence test; the student needed to: (1) make the correct choice of test, and (2) have a deep understanding of the behaviour of the given series, as the series must satisfy the hypotheses of the test for it to be used. By acting on a process conception of a mental construction via the application of a suitable convergence test, the student will display an object conception of the notion of the convergence of an infinite series. The question items given before the analysis of each response covered limits of series, with the first item checking the formal limit definition of an infinite series. This was followed by three items aimed at checking the use of methods to evaluate infinite series. The last three items checked the (series test) methods students employ to determine convergence and divergence of infinite series.

\section{Analysis and discussion of data}

The participants exhibited diverse stages of understanding on the written limit questions. The students who gave correct responses to most of the test items were ST1, ST2, ST12, ST15 and ST28, with ST2 opting not to participate in the interviews. Students ST13, ST14, ST17, ST20 and ST21 failed to give correct responses to most of the test items and ST21 opted not to participate in the interviews. Through the interviews, we developed a deeper understanding of some of the ways of thinking that underpinned students' responses to the questions. Some of the interview questions were shared with all the students and others probed students' experience with the topic. The rest of the questions were follow-ups to issues that arose in the students' written solutions or to what they indicated during the interview.

\section{Data analysis coding}

The test questions provided us with opportunities to analyse students' written responses, which gave initial clues about their understanding of limits of sequences and series. These produced qualitative data and we used a coding modified from the work of Asiala, Cottrill, Dubinsky and Schingendorf (1997) to evaluate students' responses as follows:

- A 1 was awarded for a response showing a step-by-step procedure for the solution (action level).

- A 2 was awarded for a response showing the performing of transformations mentally and prediction of outcomes (process level).

- A3 was awarded for a response that showed encapsulation of the process into a total entity, or an object, ability to perform actions on that object, and displaying the capability to decompose an object to its underlying process when the need arises (object level).

If the responses did not meet the postulates of the PGD the attempt was coded as $\mathrm{N}$ level with a zero (0) assigned.

\section{Results emanating from data analysis}

The designed tasks granted a case analysis of each test question supported by authentic written responses and interview extracts from selected students. This was done to provide confirmation of the APOS level at which the students operated, in terms of understanding the convergence of a series concept. The data presentation and analysis were done in line with the GD provided in Table 1.

Task 1a tested students' attainment of the action level conception. The results revealed that $63 \%$ of the students attained the action conception, with $37 \%$ failing to attain the action conception. Tasks $1 \mathrm{~b}$ (i) to $1 \mathrm{~b}$ (iii) tested students' attainment of the process level and the results revealed at least $63 \%$ of the students attained the process level conception. Furthermore, responses to tasks 1c (i) to 1c (iii) showed that $67 \%$ of students managed to attain the object level conception. The relevant information displayed in Table 2 was extracted for Tables 3-9 to make it easier for the reader to follow the focused discussion on the different sub-questions.

TABLE 2: Complete summary of the categorisation of students' mental construction, according to APOS, on each of the test items on limit of series.

\begin{tabular}{lccccc}
\hline Task item & \multicolumn{5}{c}{ APOS categorisation of students' mental constructions } \\
\cline { 2 - 6 } & None & Action & Process & Object & Schema \\
\hline 1a & 11 & 19 & 0 & 0 & 0 \\
1b (i) & 5 & 0 & 25 & 0 & 0 \\
1b (ii) & 10 & 0 & 20 & 0 & 0 \\
1b (iii) & 11 & 0 & 19 & 0 & 0 \\
1c (i) & 10 & 0 & 0 & 20 & 0 \\
1c (ii) & 10 & 0 & 0 & 20 & 0 \\
1c (iii) & 10 & 0 & 0 & 20 & 0 \\
\hline
\end{tabular}

APOS, action-process-object-schema.

TABLE 3: Frequency of students' responses for task 1a according to APOS level.

\begin{tabular}{llc}
\hline APOS level & $\boldsymbol{N}$ & Action \\
\hline Number of responses & 11 & 19 \\
\hline
\end{tabular}

APOS, action-process-object-schema.

TABLE 4: Frequency of students' responses for task $1 \mathrm{~b}$ (i) according to APOS level.

\begin{tabular}{lcc}
\hline APOS level & $N$ & Process \\
\hline Number of responses & 5 & 25 \\
\hline
\end{tabular}

APOS, action-process-object-schema.

TABLE 5: Frequency of students' responses for task $1 \mathrm{~b}$ (ii) according to APOS level.

\begin{tabular}{llc}
\hline APOS level & $N$ & Process \\
\hline Number of responses & 10 & 20 \\
\hline
\end{tabular}

APOS, action-process-object-schema.

TABLE 6: Frequency of students' responses for task $1 \mathrm{~b}$ (iii) according to APOS level.

\begin{tabular}{llc}
\hline APOS level & $N$ & Process \\
\hline Number of responses & 11 & 19 \\
\hline
\end{tabular}

APOS, action-process-object-schema.

TABLE 7: Frequency of students' responses for task 1c (i) according to APOS level.

\begin{tabular}{lcc}
\hline APOS level & $N$ & Object \\
\hline Number of responses & 10 & 20 \\
\hline
\end{tabular}


TABLE 8: Frequency of students' responses for task 1c (ii) according to APOS level.

\begin{tabular}{llc}
\hline APOS level & $\boldsymbol{N}$ & Object \\
\hline Number of responses & 10 & 20 \\
\hline
\end{tabular}

TABLE 9: Frequency of students' responses for task 1c (iii) according to APOS level.

\begin{tabular}{lcc}
\hline APOS level & $\boldsymbol{N}$ & Object \\
\hline Number of responses & 10 & 20 \\
\hline
\end{tabular}

\section{Discussion of data}

This being a qualitative study allowed us to analyse the 30 students' written responses and indicate the results in Table 2. For an in-depth analysis eight students were interviewed and a discussion on an exemplar for each task was presented. Each case discussed highlights the group of students falling in the category of the specific mental construction. In this subsection we provide the task given to students, a tabular summary of the results from the 30 students' written responses to questions, a written attempt depicting the case of students not displaying the particular mental construction followed by one sample of those students demonstrating an attempt in line with the dictates of the PGD. After a discussion on each written attempt, we provide an interview excerpt followed by a discussion on the interview. For each task we verified whether the findings emanating from the data analysis concurred with the PGD or not. Where there was non-alignment with our PGD we kept this in mind for the formulation of the modified GD.

\section{Task 1a: Define the limit of an infinite series}

Responses of students according to APOS levels: Nineteen $(63 \%)$ of the students gave the appropriate response to task $1 \mathrm{a}$, thus operated at the action level of the APOS theory. The responses to task 1a indicated that $11(37 \%)$ of the students could not display an action level conception according to the APOS theory, referred to as $\mathrm{N}$ level. Of the 11 students, $5 \mathrm{did}$ not attempt the question at all, while 6 gave incorrect and confusing responses.

Response at $\mathbf{N}$ level (incorrect response): The written response of ST20 (see Figure 1) does not illustrate the definition of a limit of a series. Rather he seems to be referring to what a divergent series is. This response could be due to the individual learning mathematical concepts without a clear understanding. To check how he understood these concepts, we interviewed him.

R: Can you explain the definition of the limit of an infinite series you gave?

ST20: By the time I wrote the response, I mixed up issues. I thought of the existence of the limit of a function, yet the question required the definition of an infinite series. I cannot provide the definition accurately at the moment.

This verbal response revealed that ST20 operated at N level as he failed to appropriately state the definition of the limit of an infinite series. Furthermore, his failure to state the

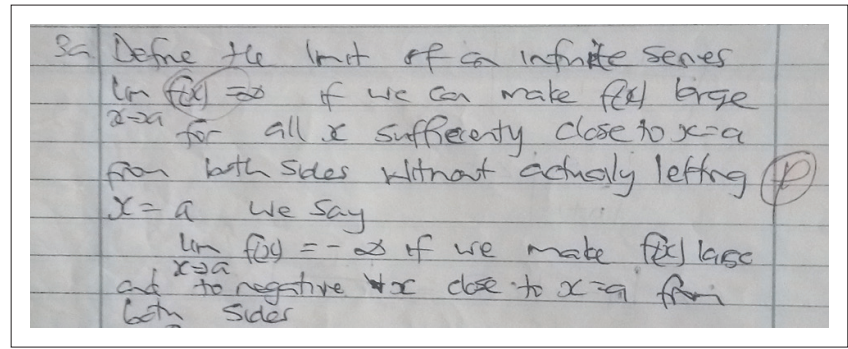

FIGURE 1: Written response of ST20 to task 1a. An example of a response of a student who operated at $N$ level.

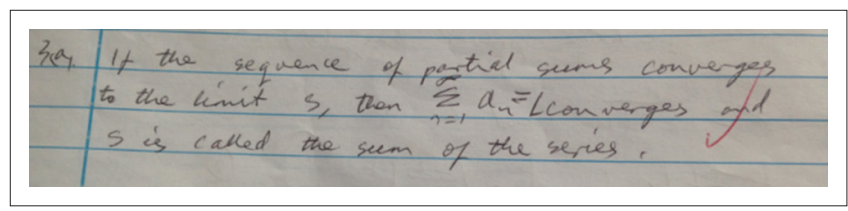

FIGURE 2: Written response of ST15 to task 1a.

definition from memory is an indication that he has not attained the action level. This question was based on our PGD to initiate the action conception of an individual's mental construction of the limit of a series concept leading to the understanding of the convergence of a series concept. However, from the response of ST20, we learn that we should provide opportunity for students to relate to the differences of convergent series and divergent ones. We kept this in mind when modifying our PGD.

Responses at an action level conception: ST15 (see Figure 2) managed to give a correct response to task 1a. Her ability to recall the definition correctly is an indication that she operated at the action level of the APOS theory. She exhibited a strong tendency to recall verbatim the definition, which is an indicator of the action level. We analysed the student's written (Figure 2) and interview responses and found that she displayed an action conception as this recall was an external manipulation. We realised that ST15 used different symbols for the limit of the partial sums and the series itself. During the interview with the student she did indicate that it should have been the same.

\section{Task 1b (i): Determination of convergence of series of $\sum_{n=1}^{\infty} n$}

Responses of students according to APOS levels: Twentyfive $(83 \%)$ of the students gave responses that showed they operated at the process level of the APOS theory. Their responses indicated they understood how to evaluate convergent series problems. Five $(17 \%)$ of the students' responses showed that those students did not attain the process level of the APOS theory. Four students out of those five substituted $n$ with infinity and concluded that the series diverges.

Responses at $\mathbf{N}$ level (totally incorrect response): The response provided by student ST17 displayed that she did not know the definition of the convergence of a series. It could be that this student used the nth term test (the test for 
divergence). The student also used the = sign incorrectly in the second line. This therefore made us place her mental constructions at the $\mathrm{N}$ level conception. One of us interviewed her to check on her understanding of what she did.

R: How did you determine that the limit of the series $1 \mathrm{~b}(\mathrm{i})$ exists?

ST17: I tried to find the last term. Then discovered that the last term goes to infinity. I am not able to determine that at the moment. It requires reading more about summation of series, maybe I have to start from advanced level work on series. It is difficult to find a formula for the general term in the sequence of partial sums.

Since ST17 did not indicate, in her responses, the definition of a convergence of a series, it would seem that she did not have an action conception of the convergence of a series. Cases of trying to find the last term of the series and the inability to find the relationship between infinity and limit diverges all pointed to failure by ST17 (see Figure 3) to attain a process conception of the convergence of a series.

Response at a process level: Figure 4 shows an example of a response of a student who operated at the process level. Such students managed to give an expression of the general term

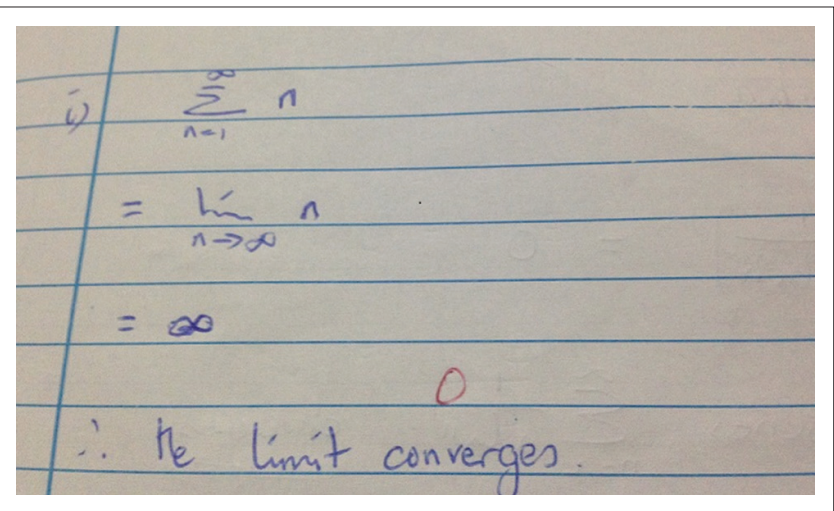

FIGURE 3: Written response of ST17 to task $1 \mathrm{~b}$ (i). An example of a response where the student operated at $\mathrm{N}$ level.

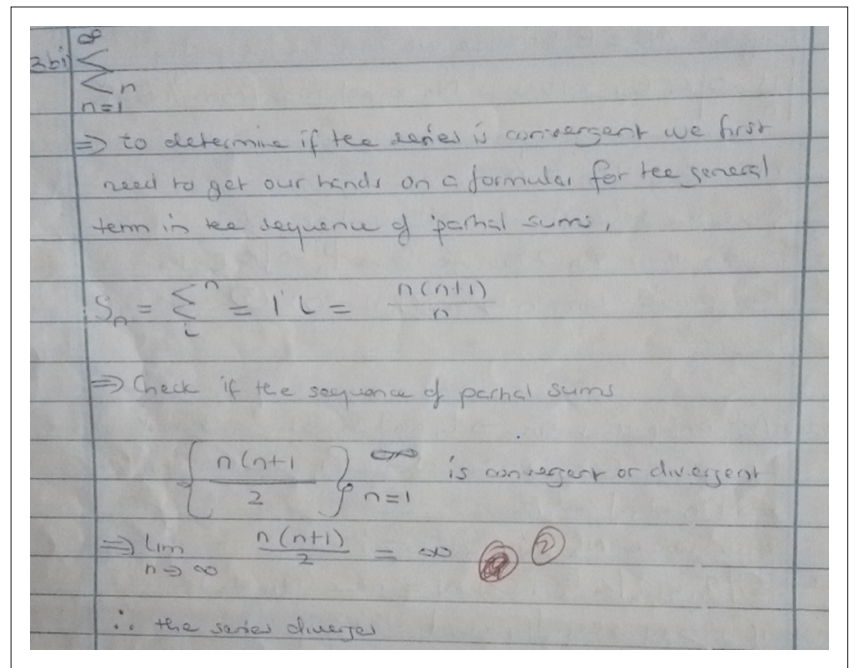

FIGURE 4: Written response of ST28 to task $1 \mathrm{~b}$ (i). for the partial sums and proceeded to show that the series diverges.

In the written response for ST28 (see Figure 4) we observe that he is applying the definition of the convergence of a series without stating it but uses the definition for this particular nth partial sum. This means that he has interiorised the action (recall of definition) into a process. ST28 was able to apply the procedure as a whole without the need to plug in specific values. To further authenticate our assertion that he operated at the process level, an interview was held to investigate strategies used.

\section{R: $\quad$ How did you find the response to task $1 \mathrm{~b}$ (i)?}

ST28: If one adds up numbers that go on and on, then one would never come to a single value. So when I checked on the given question, I saw that was the case so I had to find a formula for the general term in the sequence of partial sums. Then I borrowed the idea of finding the general expression of such a series from advanced level work. That is $\sum_{n=1}^{n} i=\frac{n(n+1)}{2}$ and the limit of the sequence terms is $\lim _{n \rightarrow \infty} \frac{n(n+1)}{2}=\infty$. Hence the sequence of partial sums diverges to $\infty$ and it can be concluded that the given series also diverges.

Here the student did not work out each partial sum to form the sequence of the partial sums meaning he had interiorised the action of recall of definition of the convergence of an infinite series. Hence the analysis of the interview responses confirmed that ST28 possessed a process conception of the convergence of the specific series in the question.

\section{Task 1b (ii): Using the definition in the determination of convergence of a series $\sum_{n=2}^{\infty} \frac{1}{n^{2}-1}$}

Responses of students according to APOS levels: Twenty $(67 \%)$ of the students gave the correct responses, which were indicative of the process level of the APOS theory. Those students managed to make use of partial fractions, and the partial sums of the given series, in their responses to this question. Furthermore, 10 (33\%) of the students' responses showed that they operated below the action level of the APOS theory. All were confused as to what they should have done to solve the problem.

Responses at $\mathrm{N}$ level (mathematically incorrect response): Figure 5 illustrates a response of a student who operated at $N$ level. Such students tried to add the resulting terms of the series, without success.

The response of ST13 in Figure 5 showed that the convergence of a series concept was not addressed, since the preliminary notions for the limit of a series were absent. The response provided by ST13 concurred with Earls (2017) who found that students used inappropriate tests to determine the convergence of series. An analysis by the researchers of ST13's written response showed that he operated at not even 


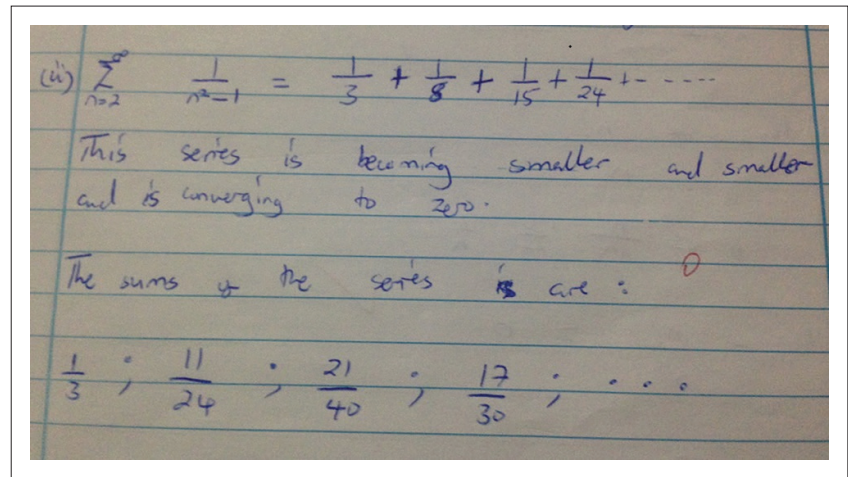

FIGURE 5: Written response of ST13 to task $1 \mathrm{~b}$ (ii).

the action level of the APOS theory. A verification of our claim was done through an interview with the student.

R: Can you give the difference between a sequence and a series?

ST13: A sequence is a list of numbers, usually in an increasing order; and a series is when these numbers are added up and their sum may or may not be found.

R: $\quad$ So how did you determine the convergence of a series given in task $1 \mathrm{~b}$ (ii)?

ST13: I substituted the values 2, 3, $4 \ldots$ into the given series expression then added the few. It was not possible to add up all the numbers to infinity as the numbers do not come to an end.

R: That is okay. Can you find the general term in the sequence of partial sums of question $1 \mathrm{~b}$ (ii)?

ST13: Um, I cannot. This topic on series was difficult for me at advanced level and still it is difficult for me.

We placed him at the $\mathrm{N}$ level (not at a process conception) after taking into consideration the responses he gave to the interview questions. Also, from the interview we observed that there was a need to consolidate differences between sequences and series. This was ignored in the PGD, so we then kept that in mind for our modified GD.

Responses at a process level: ST12 managed to give the correct response to task $1 \mathrm{~b}$ (ii) and an analysis of her response showed that she operated at the process level of the APOS theory. ST12's written response showed evidence of omission of some steps in her solution method. She also showed the application of the definition of the convergence of an infinite series which meant that she had interiorised the convergence of an infinite series concept into a process. To verify our claim an interview was conducted.

R: You gave the correct response to task $1 \mathrm{~b}$ (ii), can you take me through your response?

ST12: I thought of advanced level work where we dealt with partial fraction and then general formula for series summation. So, I found the general formula for the given series. I discovered that this results in telescoping series which resulted in $\mathrm{S}_{n}=\frac{3}{4}-\frac{1}{2 n}-\frac{1}{2 n+2}$ after some terms had canceled out. Then taking limit as $n \rightarrow \infty$ gives $\frac{3}{4}$ as the other two terms goes to zero. Thus, the sequence of partial sums converges, so the series does converge to a value of $\frac{3}{4}$.

The dialogue with ST12 verified our process mental construction placement of ST12 from the written response.

\section{Task 1b (iii): Determination of convergence of a series $\sum_{n=0}^{\infty} \frac{1}{n^{2}+3 n+2}$ by use of definition}

Responses of students according to APOS levels: Nineteen $(63 \%)$ of the students gave responses that indicted that they operated at the process level of the APOS theory. Eleven (37\%) of the students' responses showed that they were operating at the $\mathrm{N}$ level. Among the 11, one did not attempt the question, while some of the students used sequence evaluation methods.

Responses at $\mathbf{N}$ level (totally incorrect response): Figure 6 shows an example of a student who operated at the $\mathrm{N}$ level. Such a student showed inability to determine the convergence or divergence of an infinite series.

ST20 provided an incorrect response, and the method used to answer task 1b (iii) was inappropriate and did not address the question. This student failed to attain a process conception as it seemed that ST20 determined the limit of the nth term of the given infinite series instead of the partial sums. We interviewed him to check on how he understood these concepts and the associated difficulties he faced.

R: You responded to task $1 \mathrm{~b}$ (iii) as though you were answering a sequence problem. Why is this the case?

ST20: I thought since a series is an addition of numbers of a sequence, then by applying the method of evaluating

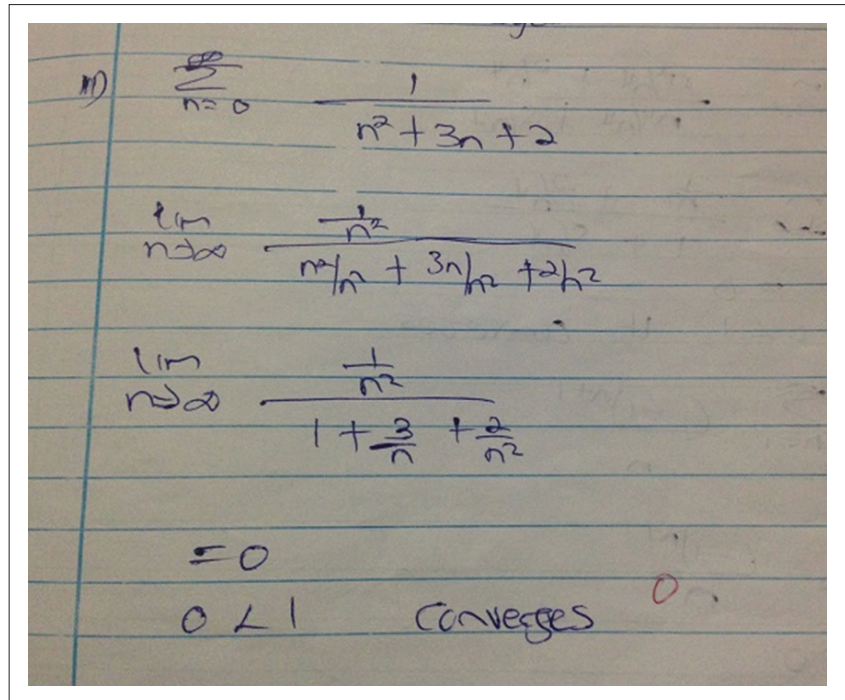

FIGURE 6: Written response of ST20 to task $1 \mathrm{~b}$ (iii). 
sequences, I would determine the convergence of the series.

R: During the learning sessions, were the convergence of a series and those of sequences determined using the same methods?

ST20: No, we did not use such methods.

R: So why did you use such a method?

ST20: I got confused during the time I was writing the test so I ended up using the wrong method.

An analysis of ST20's written response and the interview excerpts gave strong indicators that the student operated at $\mathrm{N}$ level.

Responses at a process level: Figure 7 illustrates a response of a student who operated at the process level.

Again, like the written responses of process conception attainment in the previous questions, the demonstration of the series convergence definition was pivotal in highlighting its interiorisation into a process. ST28 showed the ability to form and work with telescoping series that led him to successfully come up with the correct response. To verify ST28's understanding of these procedures and confirm whether he operated at the process level, we interviewed him.

R: You responded very well to task $1 \mathrm{~b}$ (iii), can you explain how you arrived at your final response?

ST28: Yaa, I had to think of partial fractions first, that is, expressing the given series as a sum of partial fractions. I wrote out the terms of the general partial sum for this series using partial fraction form. This resulted in a telescoping series, then I took the limit of the sequence of partial sums whose result is one as $n \rightarrow \infty$.

R: Is it always the case that successive terms will cancel out?

ST28: This is not always the case; some terms cancel with some terms way down the list. In some case instead of

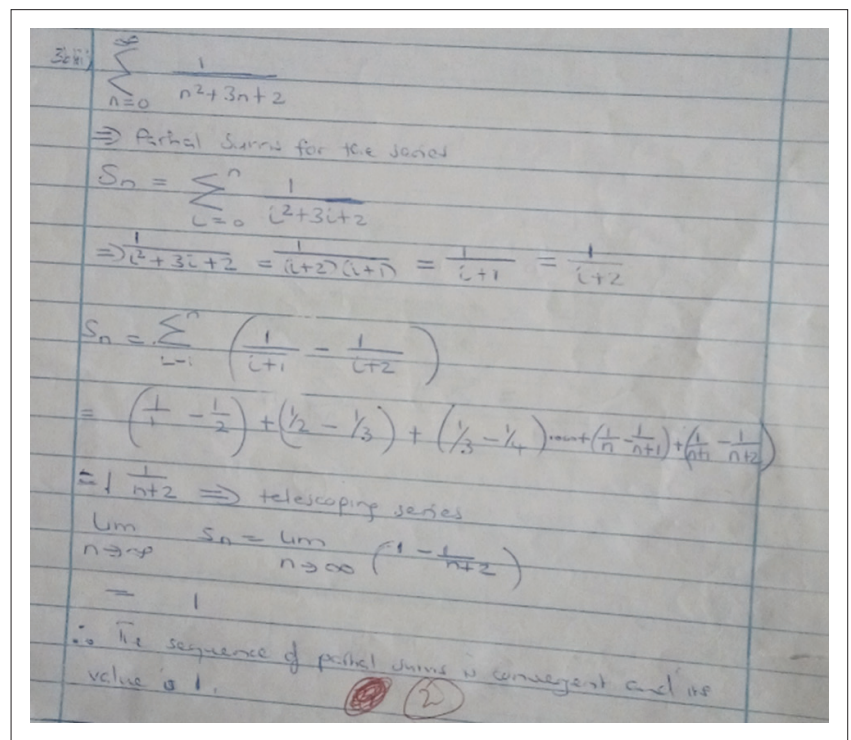

FIGURE 7: Written response of ST28 to task $1 \mathrm{~b}$ (iii). successive terms cancelling, one term will cancel with another term that is farther down the generated list. The end result this time is that the initial two and the last two terms are left.

R: What are the conditions that a series must satisfy in order for telescoping series method to be applicable to find the value of convergent series?

ST28: If we can express the series as partial fractions, have the difference of successive terms of the series, and get terms that cancel (some terms must be negative and others positive). If these three conditions are satisfied, then the telescoping series apply.

His explanations during the interview verified our placement of his mental construction at a process conception.

\section{Task 1c (i): Using a convergence/divergence test to determine convergence for $\sum_{n=2}^{\infty} \frac{1}{n \ln n}$}

Responses of students according to APOS levels: The responses to task1c (i) illustrated that 20 (67\%) students' responses showed that they operated at the object level of the APOS theory. Ten (33\%) students' responses showed that they could not attain even the action level of the APOS theory. Two students attempted the question but gave totally confused responses.

Responses at N level (no response): ST13 did not attempt question 1c (i) which is an indication that he operated at the $\mathrm{N}$ level. The student could have faced trouble with limit and series notation (Brijlall \& Ndlazi, 2019; Earls, 2017). Specifically, the student did not appear to think of the limit of a series as a limit of partial sums. We interviewed him to check his understanding of the concepts under discussion and the probable difficulties he faced.

R: $\quad$ You did not attempt task 1c (i). What challenges did you face in dealing with this question?

ST13: There are many methods of solving series limit questions for example, the integral test, ratio test, comparison series test, root test, alternating series test etc. I got confused as to which method was applicable to this particular question.

R: Do you understand the criteria for determining which method to use and when to use it?

ST13: Exactly this where I have a challenges. I need more time to work on such problems.

The student failed to make the necessary mental constructions needed to determine convergence or divergence of a series, because he failed to make the correct choice of the convergence test to be applied. He operated at the $\mathrm{N}$ level.

Responses at an object level conception: The written response in Figure 8 supported our ideas in the formulation of the PGD for the object conception of the notion of the convergence of an infinite series. ST12 was one of the students whose response showed the ability to apply the integral test 


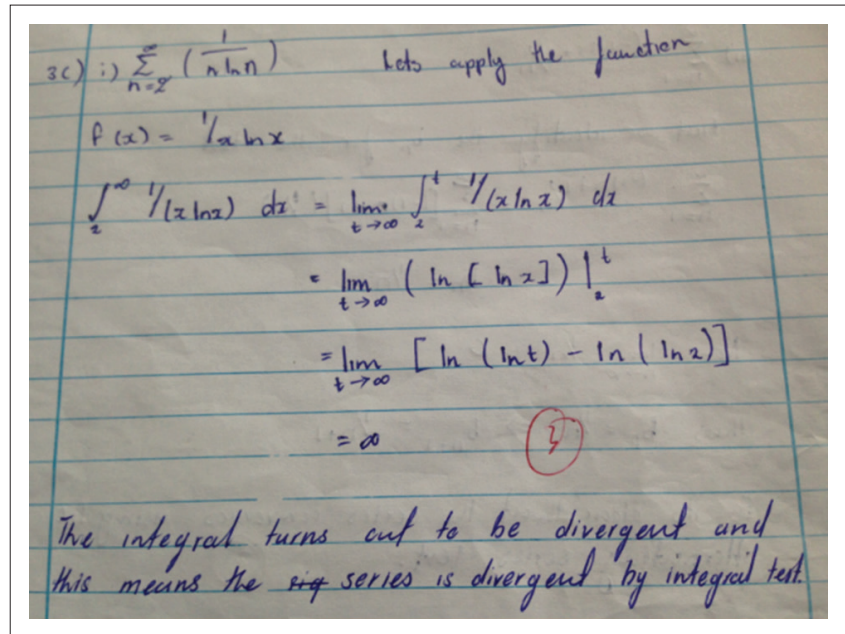

FIGURE 8: Written response of ST12 to task 1c (i).

to determine whether the series diverges. ST12 checked on the assumptions of a series test, and found that they were satisfied before using this test. To further authenticate our claim that she operated at the object level, we carried out an interview with her on her response and strategies to answer the question.

R: Given $\sum_{n=2}^{\infty} \frac{1}{n \ln (n)}$, how do you determine whether the series diverge or converge?

ST12: In this case $f(n)$ can be replaced by $f(x)$ and the result is a non-increasing continuous positive function on a given interval. If $x$ is made large, the denominator gets larger and so the function also decreases. Thus, we apply the integral test.

R: Is it possible for one to determine the value of a convergent series using the integral test?

ST12: My understanding of this series test is that it only determines whether the series converges or diverges, but does not give the value of the series because we will have replaced a sequence by a function.

ST12 demonstrated that she understood the behaviour of the given series to check whether the function is decreasing and continuous nature in the specified domain. We observe that other schemas played a role in this solution as mentioned by ST12. Reflecting on her verbal response we realised that to solve this task the student also required schemas for the concepts of increasing and decreasing functions and the notion of continuity of functions. Those we omitted in our PGD, and they aided us in the modification of our PGD. In hindsight, one shortcoming in our interview question design was that we did not gather information about the domain of integration and the reason why $n$ commenced at 2 rather than 1.

\section{Task 1c (ii): Use of a suitable test to determine the} convergence of $\sum_{n=1}^{\infty} \frac{n^{2}+2}{n^{4}+5}$

Responses of students according to APOS levels: Table 8 indicates that 20 students $(67 \%)$ gave responses that showed they operated at the object level of the APOS

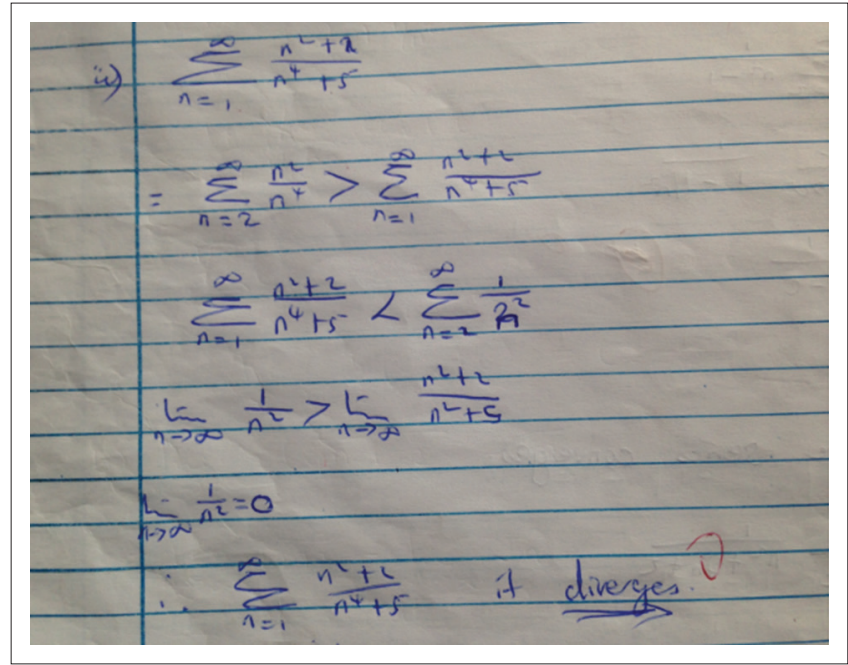

FIGURE 9: Written response of ST17 to task 1c (ii).

theory. They managed to coordinate the conditions of the comparison and $p$-series test and came up with the required solution. Ten (33\%) students did not operate even at an object conception and so were coded as operating at the $\mathrm{N}$ level.

Responses at $\mathbf{N}$ level (totally incorrect response): Figure 9 shows the response of a student who operated at the $\mathrm{N}$ level. Such responses of students showed that they had not developed an object level conception for the determination of convergence of a series.

The response given by ST17 to task 1c (ii) is a case where the student tried without success to apply the comparison test. The student displayed an inability to successfully carry out the comparison test. The student failed to check if the assumptions of the series were satisfied before using the test, which supports the finding of Earls (2017). The student failed to test if the assumptions of the direct comparison test were satisfied. ST17 operated at N level; this was revealed after we had analysed the student's written response. To confirm the accuracy of our analysis and the student's understanding of these concepts, we interviewed her.

R: Can you tell me anything you know about the comparison test for the determination of convergence of a series?

ST17: I understand that given two series, say $\sum a_{n}$ and $\sum b_{n}$ with $a_{n} \leq b_{n}$, then if the bigger is convergent then the smaller is also convergent; and if the smaller is divergent, then also the bigger one is divergent.

R: That is true. Can you explain to me how you came up with your response?

ST17: I tried to come up with two series and failed to do so. Hence the response I provided.

R: In your written response, why did you write $\lim _{n \rightarrow \infty} \frac{1}{n^{2}}=0$ ?

ST17: I wanted to, um I cannot give the reason for my response. 
R: Then how did you come up with the conclusion that the series diverges?

(the student did not respond)

An analysis of the interview with ST17 revealed that she had an action conception of the convergence of an infinite series. She managed to make the correct choice in the convergence test and dealt with the nth term of the given series, but incorrectly inserted the sigma signs. In so far as an object conception is concerned, we were correct to place her at having an $\mathrm{N}$ level understanding of the convergence of an infinite series, based on this task.

Responses at an object level conception: ST28 (see Figure 10) managed to give the correct response to question 1c (ii) and showed the ability to correctly select the most appropriate test for convergence, which he successfully carried out. Those abilities signal the possession of the object conception. The individual was able to treat that concept as a cognitive object, which incorporates the ability to perform actions on that object and give interpretation or reason about its properties (Suryadi, 2012). In order to endorse the student's knowledge of such concepts, and our claim that she operated at the object level, the interview was conducted.

R: What comment, if any, can you give about the partial sums of the infinite series?

ST28: With the series terms getting to zero in the limit, the limit might converge; but if series terms fail go to zero in the limit, then the series diverges.

R: Can you explain how you came up with the correct response to task 1c (ii)?

ST28: I made use of the terms in the infinite series, infinite process, the sum of infinite series and the links between them. These two series converge by the $p$-series test. Therefore, their sum also converges.

This student was able to display the conceptual structures indicated by our PGD in accordance with an object conception.

Task 1c (iii): Use an appropriate convergence/divergence series test to determine the convergence of $\sum_{n=1}^{\infty} \frac{(-1)^{n+1}}{n}$

Responses of students according to APOS levels: Table 9 indicates that $10(33 \%)$ of the students failed to attain an object conception when applying the alternating series test. Three of the 10 students who could not attain the object conception did provide an attempt, whereas the others did not provide any attempts. Twenty (67\%) gave responses indicative of operating at the object level, in the context of the application of the alternating series test.

Responses at N level (no response): ST14 did not attempt task 1c (iii) which was an indicator that the student operated at $\mathrm{N}$ level. We interviewed her to ascertain the difficulties she faced and how she understood these concepts.
R: You did not attempt question 1c (iii). What challenges did you face?

ST14: I found the question too difficult to answer. I need more time to work on such type of questions on the determination of convergence of series.

The excerpt above indicated that ST14 was not prepared to answer such questions. Our analysis of her responses to task 1c (iii) was also supported by her interview response. She had not made the mental constructions necessary for answering such questions and operated at $\mathrm{N}$ level.

Responses at an object level conception: Figure 11 gives an example of a student who operated at an object level. Such students showed that they had developed the necessary mental constructions to determine series convergence.

The student showed the ability to choose the appropriate convergence criteria based on the mathematical structure of task 1c (iii). A student is said have achieved object conception of a mathematical concept if the ability to treat that concept as a cognitive object is indicated, as well as the ability to carry out actions on that object, and give interpretation or reason about its properties (Suryadi, 2012). To verify his understanding of these procedures and confirm his operational level, we interviewed ST1.

R: You gave the correct response to task 1c (iii). Take me through your response.

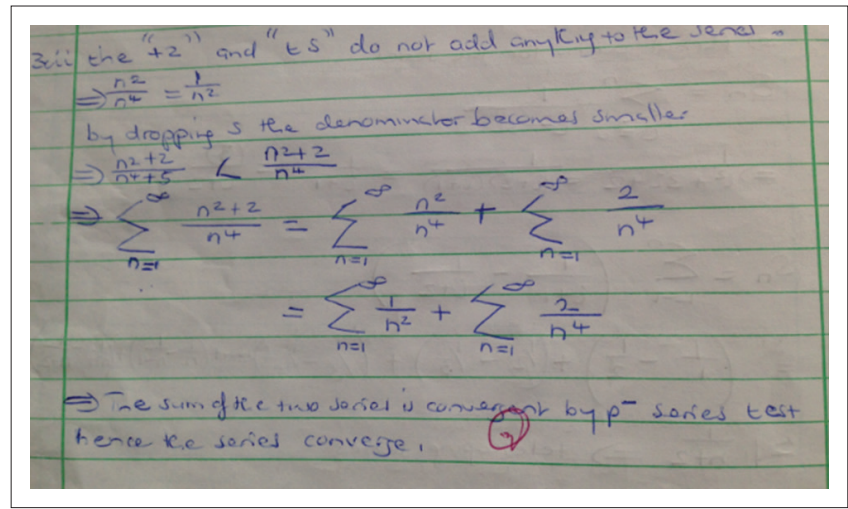

FIGURE 10: Written response of ST28 to task 1c (ii).

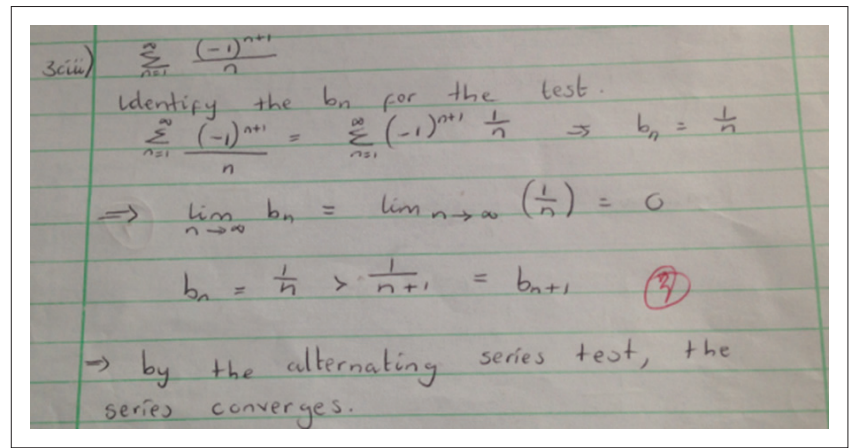

FIGURE 11: Written response of ST1 to task 1c (iii). 
ST1: This is an alternating series, so we have to carry out an alternative series test. The test is applicable if $\sum_{n=0}^{\infty}(-1)^{n} a_{n}$ is an alternating series with $a_{n}>a_{n+1}>0$ for all $n$ and $\lim _{n \rightarrow \infty} a_{n}=0$, then this series converges.

R: Can you explain how an infinite series (sequence of partial sums) has its limit as a real number?

ST1: If the infinite series converges, then its limit is a real number. One has to view an infinite process as one (a total and complete process) which we can arrive at through the use of a formula or convergence tests.

The interview confirmed that ST1 operated at the object level conception as indicated in the PGD.

\section{Conclusion}

This article is based on research in which we studied 30 participants' written responses to questions. For the purposes of triangulation interviews were conducted with eight of those participants involving tasks leading to them demonstrating their mental constructions both in written and verbal responses. In the discussion an exemplar for each task was presented. Each case discussed highlighted the group of students falling in the category of the specific mental construction, supported by the relevant interview extracts. Those discussions are relevant only for the eight students that were interviewed and cannot be generalised to the entire group of 30 participants. Also, in this study we explored whether the students for a particular task either demonstrated a particular mental construction or not. This approach showed shortcomings so for future studies it will be necessary that for a full understanding of a mathematical concept, a student needs to exhibit all 'levels' or rather categories simultaneously.

For the limits of series there was one task aimed at the action level. Students' responses matched some past research studies. The following conclusions are made from the tables in the discussion of data section. The information summarised in those tables was arrived at from an analysis of only the 30 participants' written responses to the questions. In answering the first research question on what mental constructions undergraduate mathematics students reveal when solving problems involving the convergence of a series concept, we found that 19 out of 30 participants managed to attain an action conception of defining the limit of an infinite series. Furthermore, the tasks on the definition of convergence of an infinite series $1 \mathrm{~b}$ (i-iii) on the limit of the sequence of partial sums revealed that 25,20 and 19 students displayed a process conception. The students' response to tasks 1c (i-iii) revealed that 20 students operated at the object level conception, in each case. Note that many of those 20 students overlapped with the students who displayed a process conception for tasks $1 \mathrm{~b}$ (i-iii). We also noted that a participant could be at the object level for one example of an infinite series, but at the $\mathrm{N}$ level for another example of an infinite series. For such observations from the data we believe there is a need for another study with this as a focus, which was outside the scope of the present study.

During the analysis of the data and findings therefrom we observed that our PGD did not take into account: (1) at least an action conception of sequences and series with a purpose to display mental constructions which will accommodate for their differences, (2) at least an action conception of
TABLE 10: Preliminary and modified genetic decompositions for series. Preliminary genetic decomposition

Action conception of a series by demonstrating examples of series, stating the definition of the limit of an infinite series and recall of the definition of convergence of a series.

Process conception: The individual is able to find the solution of a given question on limit of series without carrying out step-by-step procedures. At this level, the individual is able to predict whether a series converges or diverges by looking at its structure, and use properties of series to evaluate limits of series by showing the

ability to coordinate actions to come up with responses, for example to find the limit of the series $\sum_{n=2}^{\infty} \frac{1}{n^{2}-1}$

Object conception: The individual is able to determine convergence or divergence of series using series tests without detailed explanations, for example finding the limit of the series $\sum_{n=1}^{\infty} \frac{n^{2}+2}{n^{4}+5}$. Actions are performed on a process, the subject must encapsulate it to become a total entity, or an object. A student is capable of treating a limit of a series concept as a cognitive object which comprises the ability to do action on that object, and give an explanation or reason about its properties. For instance, a student should be able to demonstrate the convergence of the sum of two convergent infinite series.

Schema attainment: The individual shows the ability to verify all the conditions necessary for convergence when using the series test, make illustrations of the given task, evaluate the integral and give an appropriate conclusion in a coherent framework. The individual is expected to show coherence by making use of appropriate schema to use the integral test to determine the convergence of $\sum_{n=2}^{\infty} \frac{1}{n \ln n}$, with a detailed explanation of the working.

\section{Modified genetic decomposition}

Action conception of a series by demonstrating examples of series, stating the definition of the limit of an infinite series and recall of the definition of convergence of a series. Here the students see the limit of the series by actually adding up terms of the series and forming partial sums.

Action conception of infinite sequences.

Action conception of infinite sequences and infinite series to demonstrate their differences.

Action conception of the concepts of convergence and divergence of an infinite series to demonstrate their differences.

The action is interiorised into a process when the individual can quickly find the result of limit of series by imagining without carrying out all steps. The individual realises that the adding action can and will continue without end, and has no need to actually add any terms This process conception can be viewed when the student deals with:

1. Partial fractions decomposition to determine limits of series.

2. Application of telescoping series to determine limits of series.

3. Increasing or decreasing functions.

4. Continuity of functions.

Object conception of the notion of convergence of an infinite series. When the above processes are encapsulated into an object which is demonstrated when the individual:

1. Examines if given expressions (series test question) satisfy the appropriate hypotheses of the required particular convergence tests. Acts upon a process conception of the continuity notion or acts upon the process conception of a monotonic increasing function notion.

2. These actions on processes are conceptualised into an object conception.

3. Makes the correct choice of series test to be used to determine convergence or divergence of given series.

4. Applies conditions and properties of series tests to determine convergence or divergence of given series.

The schema level is attained when the individual is able to:

1. Show evidence of the collection of the above actions, processes, objects and other schemas in a coherent structure.

2. State and use the conditions of series tests and use graphical and analytical registers jointly to determine convergence of series. 
convergence and divergence of series with a purpose to display mental constructions which will accommodate for their differences, (3) an action or process conception of resolving rational functions into partial fractions, (4) at least a process conception of the increasing and decreasing function notions, and (5) a process conception of the notion of continuity of functions. Based on these shortcomings we made revisions and present a modified GD (which addresses research question two) in Table 10. This modified GD could be used by mathematics educators to design questions for instruction, consolidation and assessment. The modified DG gives an insight into the types of mental constructions that need to be focused on during the teaching and learning process.

\section{Acknowledgements Competing interests}

The authors have declared that no competing interest exists.

\section{Authors' contributions}

All authors contributed equally to this work.

\section{Ethical consideration}

Ethical clearance was received by the University of KwaZuluNatal (number HSS/0953/016D). Approval was also received by Bindura University.

\section{Funding information}

This research received no specific grant from any funding agency in the public, commercial or not-for-profit sectors.

\section{Data availability statement}

The data are from the corresponding author's $\mathrm{PhD}$ thesis.

\section{Disclaimer}

The views and opinions expressed in this article are those of the authors and do not necessarily reflect the official policy or position of any affiliated agency of the authors.

\section{References}

Arnon, I., Cottrill, J., Dubinsky, E., Oktac, A., Trigueros, S., \& Weller, K. (2014). A framework for research and curriculum development in mathematics education New York, NY: Springer.

Asiala, M., Cottrill, J., Dubinsky, E., \& Schingendorf, K.E. (1997). The development of students' graphical understanding of the derivative. Journal of Mathematical Behaviour, 16(4), 399-431. https://doi.org/10.1016/S0732-3123(97)90015-8

Aydin, S., \& Mutlu, C. (2013). Students' understanding of the concept of limit of a function in vocational high school mathematics. The Online Journal of Science and Technology, 3(1), 145-152.

Bansilal, S., Brijlall, D., \& Trigueros, M. (2017) An APOS study on pre-service teachers' understanding of injections and surjections. Journal of Mathematical Behavior 48, 22-37. https://doi.org/10.1016/j.jmathb.2017.08.002

Bitsch, V. (2005). Qualitative research: A grounded theory example and evaluation criteria. Journal of Agribusiness, 23(1), 75-91.

Brijlall, D., \& Ndlazi, N.J. (2019). Analysing engineering students' understanding of integration A to propose a genetic decomposition. Journal of Mathematical Behaviour. https://doi.org/10.1016/j.jmathb.2019.01.006.

Cappetta, R., \& Zollman, A. (2013). Agents of change in promoting reflective abstraction: A quasi-experimental, study on limits in college calculus. Journal of Research in Mathematics Education, 2(3), 343-357.

Chagwia. C.J. (2020). Exploring University Students' mental constructions of the Limit concept in relation to Sequences and Series (Unpublished doctoral dissertation). University of KwaZulu-Natal, Durban.

Dubinsky, E., \& McDonald, M. (2002). APOS: A constructivist theory of learning in undergraduate mathematics education research. Retrieved from http://www. undergraduate mathematics education research. Retriev
math.wisc.edu/ $\sim$ wilson/Courses/Math903/ICMIPAPE.PDF

Denbel, D. (2014). Students' misconceptions of the limit concept in a first Calculus course. Journal of Education and Practice, 5(34), 24-40.

Earls, D. (2017). Student's misconceptions of sequences and series in second semester calculus. Doctoral Dissertations. 156. Durham: University of New Hampshire. Retrieved from https://scholars.unh.edu/dissertation/156

Earls, D., \& Demeke, E. (2016). Does it converge? A look at second semester calculus students' struggles determining convergence of series. Proceeding for the XIX Annual Conference on Research in Undergraduate mathematics Education (RUME) (pp. 704-710). Pittsburgh, PA, 25-27 February.

Gulcer, B. (2012). Examining the discourse on the limit concept in a beginning-level calculus clssrrom. Educational Studies in Mathematics, 82(3), 439-453. https:// doi.org/10.1007/s10649-012-9438-2

Hartman. J. (2008). Infinite series in calculus. Columbus, $\mathrm{OH}$ : College Board.

Joseph, G.G. (2000). The crest of the peacock: Non-European roots of mathematics. Priceton, NJ: Princeton University Press.

Martínez-Planell, R., Gonzalez, A.C., DiCristina, G., \& Acevedo, V. (2012). Students' conception of infinite series. Educational Studies in Mathematics, 81(2), 235-249. https://doi.org/10.1007/s10649-012-9401-2

McCombs, P. (2014). Analysis of second semester calculus students' understanding of series and series convergence. DeKalb, IL: Northern Illinois University.

Nardi, E., \& lannone, P. (2001). On convergence of a series: The unbearable inconclusiveness of the limit comparison test. In M. van den Heuvel-Panhuizen (ed.), Proceedings of the 25th Conference of the International Group of the Psychology of Mathematics Education (PME) (pp. 399-406). Utrecht: PME.

Patel, R., McCombs, P., \& Zollman, A. (2014). Metaphor clusters: A study characterizing instructor metaphorical reasoning on limit concept in calculus. School Science and Mathematics, 114(5), 236-245. https://doi.org/10.1111/ssm.12072

Samkova, L., (2012). Calculus of one and more variables with Maple. Internationa Jourmal Mathematics Education Science and Technology, 43(2), 230-244. https:// doi.org/10.1080/0020739X.2011.582248

Stillwell, J. (1989). Mathematics and its history. New York, NY: Springer-Verlag. https:// doi.org/10.1007/978-1-4899-0007-4

Suryadi, D. (2012). Building a new culture in mathematical thinking. Bandung: Rizqi Press.

Wahyuni, D. (2012). The research design maze: Understanding paradigms, cases, methods and methodologies. Journal of Applied Management Accounting Research, 10(1), 69-80. 